\author{
Available online at www.sciencedirect.com \\ science@directs
}

Archives

of

Oral

ELSEVIER

\section{Biology}

www.intl.elsevierhealth.com/journals/arob

\title{
Heterogeneous activation of the medial pterygoid muscle during simulated clenching
}

\author{
Hans J. Schindler ${ }^{a, c, *}$, Stefan Rues ${ }^{a}$, Jens C. Türp ${ }^{b, d}$, Jürgen Lenz ${ }^{a}$ \\ ${ }^{a}$ Research Group Biomechanics, Faculty for Mathematics, University of Karlsruhe, Germany \\ ${ }^{\mathrm{b}}$ Department of Reconstructive Dentistry and Temporomandibular Disorders, Dental School, \\ University of Basel, Switzerland \\ ${ }^{\mathrm{C}}$ Federal Research Center for Nutrition, Karlsruhe, Germany \\ ${ }^{d}$ Department of Prosthodontics, Dental School, University Hospital Freiburg, Germany
}

Accepted 24 November 2005

\section{KEYWORDS}

Masticatory muscles;

Heterogeneous muscle

activation;

Clenching force;

Electromyography
Summary The aim of this study was to investigate whether the medial pterygoid muscle shows differential activation under experimental conditions simulating force generation during jaw clenching.

To answer this question, the electromyographic activity of the right medial pterygoid was recorded with two intramuscular electrodes placed in an anterior and posterior muscle region, respectively. Intraoral force transfer and force measurement were achieved by a central bearing pin device equipped with strain gauges. The activity distribution in the muscle was recorded in a central mandibular position during generation of eight different force vectors at a constant amount of force $(F=150 \mathrm{~N})$.

The investigated muscle regions showed different amounts of EMG activity. The relative intensity of the activation in the two regions changed depending on the task. In other words, the muscle regions demonstrated heterogeneous changes of the EMG pattern for various motor tasks.

The results indicate a heterogeneous activation of the medial pterygoid muscle under test conditions simulating force generation during clenching. This muscle behaviour might offer an explanatory model for the therapeutic effects of oral splints. (C) 2005 Elsevier Ltd. All rights reserved.

\section{Introduction}

Jaw muscles are traditionally considered as a homogeneous unit. This corresponds to the idea that all

\footnotetext{
* Corresponding author. Tel.: +49 721 9333713; fax: +49 721358546.

E-mail address: myo.schindler@t-online.de (H.J. Schindler).
}

motoneurons of a specific muscle receive the same synaptic input ${ }^{1}$ and that they are activated in a predetermined order corresponding to their cell size. ${ }^{2}$ Recent findings show, however, that - similar to some muscles in the extremities ${ }^{3-5}$ - the jaw musculature is capable of differential, i.e., heterogeneous, activation. ${ }^{6-10}$ As a result, regions of an individual muscle demonstrate distinct differences 
in their activation relative to one another ${ }^{11}$ when various motor tasks are performed. In contrast to the 'mosaic pattern' in the extremities with fibre distribution throughout large areas of the muscle cross-section, ${ }^{12}$ the more focal distribution of the motor units in the jaw muscles ${ }^{13}$ as well as the regionally heterogeneous histochemical fibre profile ${ }^{14}$ point to local functional differences. Additionally, the heterogeneous distribution of muscle spindles as found in the masseter muscle $e^{15,16}$ suggests a special role for certain muscle regions in the control of a particular motor task. Apart from the implications for biomechanical modelling, such data are of special clinical interest, because the regional differences in the jaw muscle activation might give a deeper insight into pathophysiological mechanisms which are supposed to be responsible for regional muscle pain caused by overload. ${ }^{17-20}$ Thus far, the phenomenon of differential activation has been substantiated for the masseter, ${ }^{8,21}$ the lateral pterygoid, ${ }^{22}$ and the digastric muscles. ${ }^{23}$ Evidence for the temporalis is conflicting, because the heterogeneous activation has been demonstrated by some groups, ${ }^{7}$ but not by others. ${ }^{24}$ The goal of this study was to investigate if a differential activation can also be detected for the medial pterygoid.

\section{Materials and methods}

\section{Subjects}

Ten healthy male subjects (average age: $29 \pm 2.6$ years) took part in the experiments. The subjects had Angle class I or mild class II dentitions. Exclusion criteria were skeletal anomalies (e.g., short-faced or long-faced) or distinct malocclusions. The study was approved by the Ethics Committee of the University of Freiburg, Germany (No. 25/02). All participating subjects gave their written consent to the experiments, which were conducted in accordance with the Declaration of Helsinki.

\section{Intraoral force simulation and force measurement}

The methods of intraoral force simulation and force measurement were described in detail in a recent publication. ${ }^{10}$ Briefly, the force was centrally transmitted by an intraoral bearing pin device, which was equipped with strain gauges and fixed on custom made metal splints. For individual adjustment of the measuring device, the casts of the subjects were mounted in maximum intercuspation in an articulator. The lower plate was mounted parallel to the occlusal plane of the mandible, and the base plate of the maxillary bearing pin was positioned parallel to the lower contact plate so that the pin met the intersection point of a connecting line between the mesial border of the lower first molars and the midsagittal plane at a right angle (Fig. 1). Jaw separation as measured at the incisor region was adjusted to $5 \mathrm{~mm}$ for each subject. The intersection between the contact plate and the midsagittal plane was selected as the $x$-axis of the measuring device (Fig. 1). Intraoral gothic arch recordings served to define central jaw position on the contact plate of the mandible. The central position was marked by a hole. The perforation enabled a joint connection with a loose fit between the tip of the maxillary bearing pin and the lower jaw plate, and it ensured the simulation of bilateral clenching in vertical and horizontal directions. The transducer allowed measurement of forces in three orthogonal directions (anteroposterior $=x$-axis, left - right $=y$ axis, vertical $=z$-axis). The signals were amplified and displayed on a monitor. The signals were digitised (sampling rate: $2000 \mathrm{~Hz}$ ) synchronously to the EMG signals.

\section{Feedback}

The target force vector was displayed to the subjects on a monitor. ${ }^{25}$ Angle $\varphi$ (angle between the $x$-axis and the projection of the force vector onto the $x, y$-plane) and angle $\theta$ (angle between the $z$-axis and the force vector) were displayed in a planar coordinate system as a vector (Fig. 2). Angle $\varphi$ was plotted in the circumferential and angle $\theta$ in the

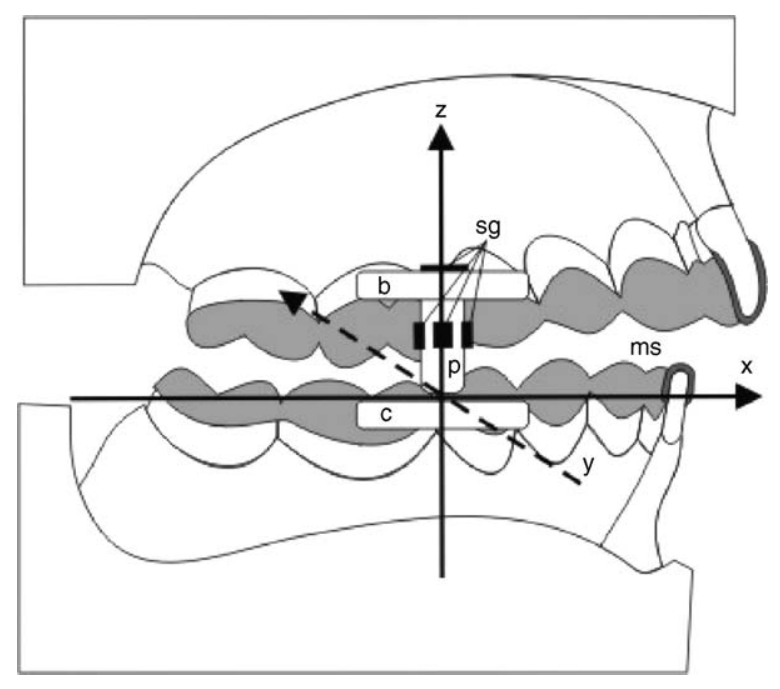

Figure 1 Sketch of the incorporated force transducer and its orientation in the Cartesian $(x, y, z)$ coordinate system. sg: strain gauges, p: pin; ms: metal splint, c: contact plate; b: base plate. 


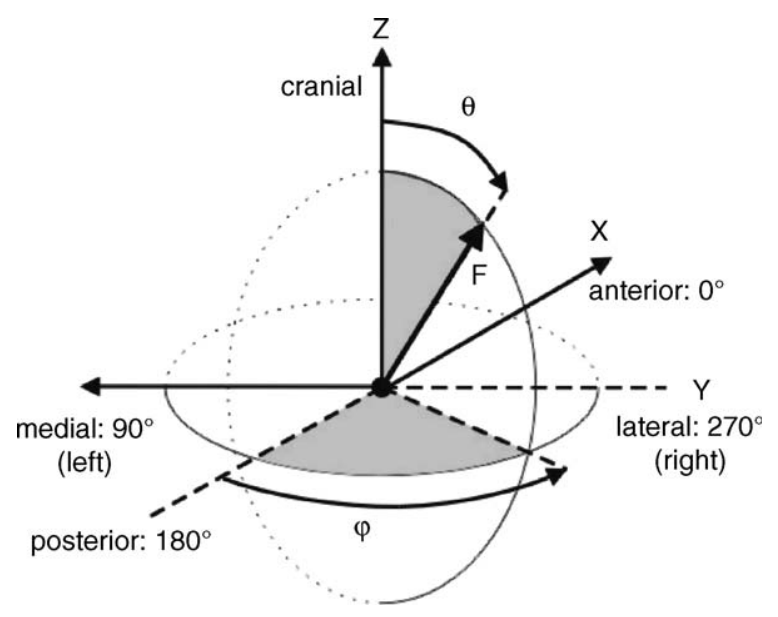

Figure 2 Angles $\theta$ and $\varphi$ in the used spherical coordinate system; $F$ : resultant force.

radial direction. The amount of force was shown on the display as an additional vertical bar with scaling.

\section{EMG recordings}

Bipolar wire electrodes recorded the electrical activity of the right medial pterygoid in a posterior and anterior region. Each electrode consisted of two Teflon-coated wires made of stainless steel (diameter $0.08 \mathrm{~mm}$; California Fine Wire, Grover Beach, CA, USA) with de-insulated ends $(2 \mathrm{~mm})$ that were bent to a hook. The electrodes were inserted using $0.4 \mathrm{~mm} \times 40 \mathrm{~mm}$ disposable needles. An extraoral approach was used to gain access to the medial pterygoid. The penetration parameters were inferred from available magnetic resonance tomograms of the 10 test persons. Horizontal slices parallel to the Frankfort plane allowed the estimation of the anteroposterior extension of the muscle. Coronal slices were used to determine the penetration depth and the angulation between origin and insertion of the medial pterygoid as described below.

\section{Insertion in the medial pterygoid}

A custom-made template was made of a rectangular metal plate bent to an angle of $135^{\circ}$ (Fig. 3). The device was aligned to the mandible in such a way that the inclined posterior part (length $30 \mathrm{~mm}$; width $7 \mathrm{~mm}$; thickness $2 \mathrm{~mm}$ ) rested against the ascending ramus, while the horizontal part (length $40 \mathrm{~mm}$; width $20 \mathrm{~mm}$; thickness $2 \mathrm{~mm}$ ) was in close contact with the lower border of the mandibular body (Fig. 3). The template was used to displace the submandibular gland medially and to force the facial artery forward before insertion of the needles. The horizontal part of the template had two prefabricated holes (diameter: $2 \mathrm{~mm}$ ) which served as insertion guides for the needles. Through the two

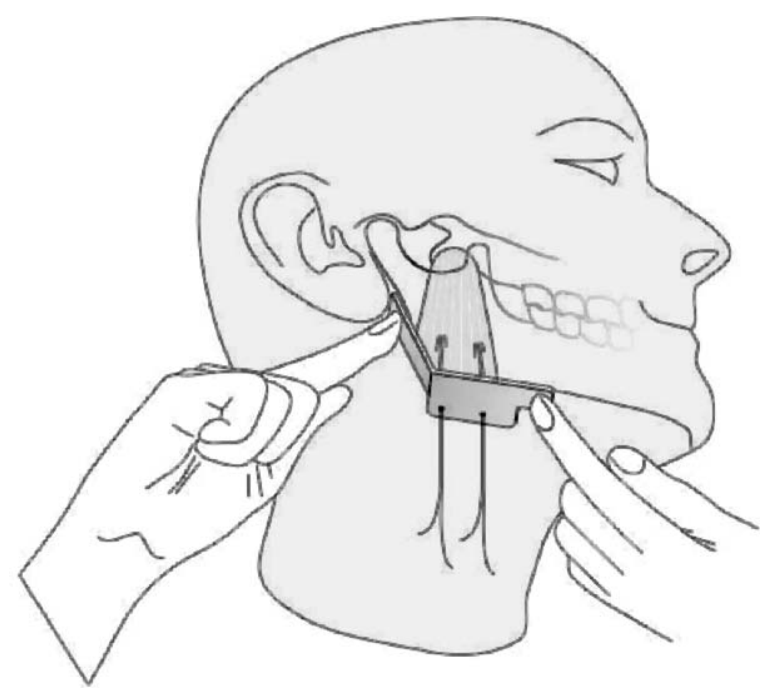

Figure 3 Sketch of the template used for standardized placement of the wire electrodes.

holes of the horizontal part, which were located 5 and $20 \mathrm{~mm}$ from the distal border and $5 \mathrm{~mm}$ from the medial rim of the plate, the needles were inserted at the medial side of the angle of the mandible in a cranially slightly medial direction (ca. $15^{\circ}$ tilted to the sagittal plane) and roughly parallel to the anterior border of the masseter muscle. For that purpose, before insertion, these landmarks were marked on the cheek with a pencil. The needles were inserted to a depth of approximately $30 \mathrm{~mm}$ and carefully withdrawn. The shortened security caps of the disposable needles were used as a penetration stop. The common electrode was positioned in the neck above the seventh vertebra. The EMG signals were differentially amplified (EM 100 Biopac, Santa Barbara, CA, USA; frequency response $1-5000 \mathrm{~Hz}$ ), and sampled at $2000 \mathrm{~Hz}$.

\section{Experimental protocol}

At the beginning of the examination, the force transducer was mounted on both jaws using zinc oxide cement (Nogenol; GC America, Alsip, IL, USA). The electrodes were then attached in the manner described above. After a few preliminary tests, which were intended to familiarize the subjects with the device and the test conditions, various motor tasks were performed in random order: Force vectors of constant magnitude $F=150 \mathrm{~N}$ were produced in the central jaw position at eight different angles $\varphi$ (anteriorly: $0^{\circ}$, anteromedially: $45^{\circ}$, medially: $90^{\circ}$, posteromedially: $135^{\circ}$, posteriorly: $180^{\circ}$, posterolaterally: $225^{\circ}$, laterally: $270^{\circ}$, anterolaterally: $315^{\circ}$ ) at a fixed angle $\theta=20^{\circ}$ (tilt to the vertical). The recording sequences were repeated three times. After the experiments, the subjects 
bit three times at maximum force in maximum intercuspation to achieve maximum activation of the medial pterygoid muscle. This test was repeated three times. To produce maximum force, the subjects received repeated vocal encouragement.

\section{Analysis of EMG data}

Using specially developed software, the point in time was determined at which the test person was closest to the given force vector, i.e., at which the error $e=\left|\vec{F}_{\text {measured }}-\vec{F}_{\text {target }}\right| /\left|\vec{F}_{\text {target }}\right|$ was minimal. An interval of $400 \mathrm{~ms}$ around this point was employed for the analysis. The obtained EMG data were rectified with the root mean square algorithm (RMS) and normalised with the maximum EMG activity found during the maximum effort tests (MVC\%).

\section{Statistical analysis}

The interindividual distribution of the measured parameters under the various conditions was described by the mean values (mean) and standard deviations (S.D.S) of the normalised data for the muscles. Additionally, the best and worst fitting case compared with the averaged data of the sample were depicted, i.e., individual cases which showed for the different tasks the least or most frequent reversals of the relative activation ratio of the two regions in comparison with the activation ratio of the means (for instance, individual ratio: anterior region more active than posterior region; ratio of the means: posterior region more active than anterior region). The intra-individual scatters of the electromyographic values for the same tasks were clarified using the coefficient of variation (CV). The minimal error of the measured force vector was determined for all subjects and all tests. The values were averaged over the 10 subjects and specified in percent (\%). The influence of the motor tasks and of the various muscle regions (eight motor tasks, two regions) on the muscle activation was examined with a two-way analysis of variance (ANOVA) with repeated measurements in both factors.

\section{Results}

The EMG activities recorded from the two muscle regions exhibited clear activation differences for the eight examined motor tasks (Fig. 4); Fig. 5 depicts a series of representative processed EMG data. Two-way ANOVA for the values measured in a central jaw position showed a significant influence of the motor tasks ( $p<0.001)$ but not by the muscle regions ( $p>0.05$ ) on the EMG activity. In addition, a significant interaction $(p<0.001)$ between the two parameters was apparent.

The most distinct activity fluctuations in EMG activity for the motor tasks between the anterior and posterior regions occurred in the anterior, anteromedial, medial, posterolateral, and anterolateral directions. During anterior and anterolateral clenching, the posterior part showed more activity. In the anteromedial, medial, and posterolateral directions the anterior region was the most active muscle part (Fig. 4). However, the individual data show that even in the best fitting case deviations from this general pattern could be observed (Fig. 4).

The mean relative activation differences between the two regions were $17.8 \pm 11.4 \%$ (range: $1.8-35.2 \%)$. The mean intra-individual variability (CV) of the EMG data for three measurement repetitions amounted to $22.1 \pm 11.3 \%$. The mean deviation of the measured force vectors from the target force vectors was $3.7 \pm 2.8 \%$.

\section{Discussion}

\section{Limitations}

Some sources of error may have affected the study: (1) It is known that the masticatory musculature may generate more than two differential activation states within an individual muscle. ${ }^{10,26}$ This might have led to the registration of different motor unit subpopulations in the two muscle regions of the

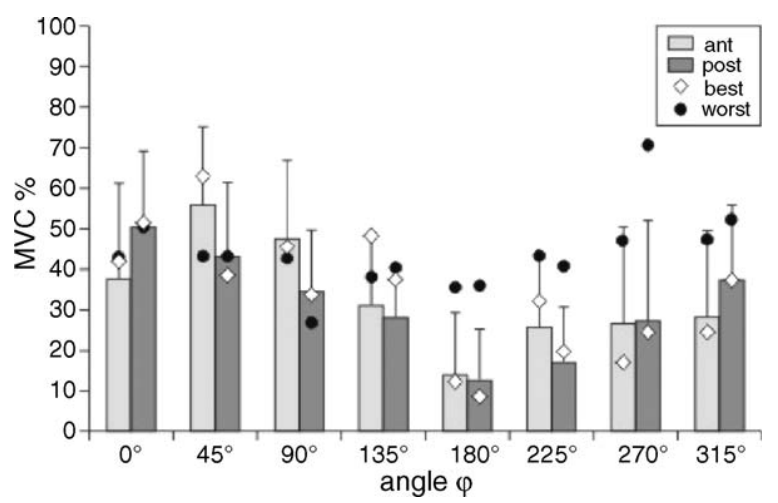

Figure 4 EMG activities (MVC \%) in the anterior and posterior muscle region at a constant muscle force $F=150 \mathrm{~N}$ and at a constant inclination of the resultant force vector to the vertical (angle $\theta=20^{\circ}$ ). Horizontal force directions: anterior $\left(\varphi=0^{\circ}\right)$, anteromedial $\left(\varphi=45^{\circ}\right)$, medial $\left(\varphi=90^{\circ}\right)$, posteromedial $\left(\varphi=135^{\circ}\right)$, posterior $\left(\varphi=180^{\circ}\right)$, posterolateral $\left(\varphi=225^{\circ}\right)$, lateral $\left(\varphi=270^{\circ}\right)$, anterolateral $\left(\varphi=315^{\circ}\right)$. Ant: anterior muscle region, post: posterior muscle region, best: best fitting case, worst: worst fitting case. 


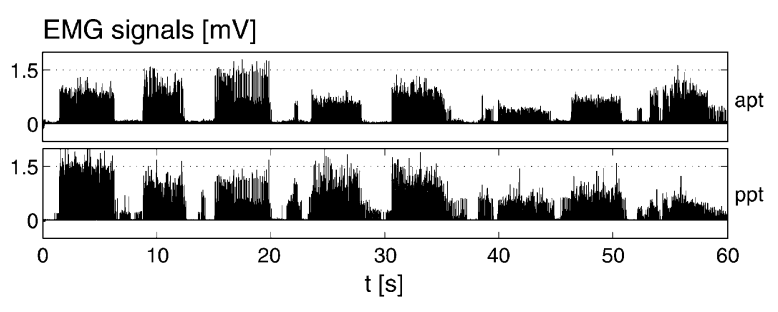

Force signals $[\mathrm{N}]$

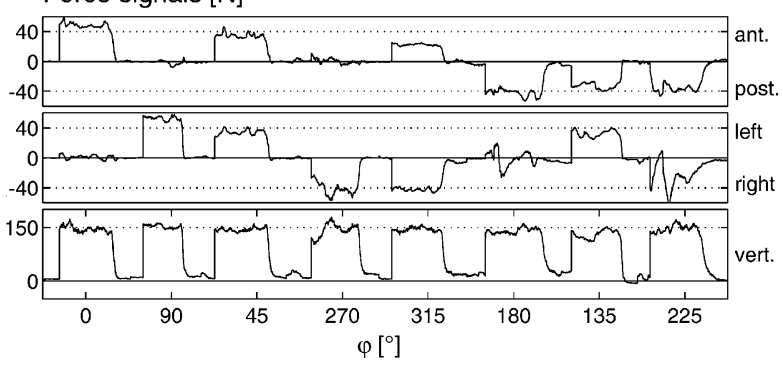

Figure 5 Sequence of rectified EMG signals of the anterior and posterior muscle regions and the corresponding force signals generated during the various clenching tasks. Apt: anterior medial pterygoid; ppt: posterior medial pterygoid; ant.: anterior; post.: posterior; vert.: vertical.

subjects. This possible source of error cannot be entirely excluded. Due to the small pick-up area of wire electrodes, it depends much on the precision of the wire insertion to record identical muscle subregions in different subjects. (2) It is possible that the measured activation differences of the electric signals were contaminated by crosstalk from adjacent regions. ${ }^{27}$ Wire electrodes exhibit a relatively high selectivity; they essentially register active muscle fibres located in the immediate vicinity of the electrodes. Due to volume conduction, however, they also record electric activities of adjacent neuromuscular compartments. ${ }^{27}$ Yet, in contrast to limb muscles with relative large neuromuscular compartments, the compartments of muscles such as the masseter are very localized; each neuromuscular compartment occupies a small restricted subvolume of the muscle ${ }^{28}$ with a mean diameter of the motor unit territory of about $3.7 \pm 2.3 \mathrm{~mm}$ in medio-lateral direction. ${ }^{29}$ The masseter and the medial pterygoid muscles demonstrate close structural similarities. Hence, it seems to be justified to suppose a similar arrangement of the motor units. Therefore, in view of interelectrode distances of approximately $15 \mathrm{~mm}$ as in our study, it is doubtful that the intramuscular electrodes registered considerable crosstalk from adjacent compartments. (3) Another restriction in our experimental model was that the force transfer was managed midsagittally on the level of the second premolars. These specific factors limit the direct comparison with activation states in maximum intercuspation. How- ever, the good match of the mean EMG data during maximum contraction with and without the bearing pin device as shown for the masseter ${ }^{10}$ and all other masticatory muscles ${ }^{30}$ speaks for a similar stiffening behaviour of the system in the two positions, i.e., the neuromuscular system balances the muscle forces and joint reaction forces in a similar way to reach static equilibrium. This leads us to assume that our results represent a good approximation of clenching activities that occur in maximum intercuspation.

\section{Data variability}

The intra-individual EMG variability (CV) for repetitive motor tasks amounted on the average to $22.1 \pm 11.3 \%$. Deviations in force direction and in the amount of force during performance of the motor tasks are possible explanations for this scatter. Another factor to be taken into account is that the musculature of individuals may generate slightly different recruitment patterns to perform the same motor tasks. Among other possible reasons, these activity variations could either represent a normal behaviour or they could be the result of a compromised periodontal proprioception. It is well known that mechanoreceptors in the periodontal ligament encode the magnitude and direction of the bite force. ${ }^{31}$ Fixation of all teeth with metal splints may have reduced this capacity, which might have negatively influenced the precision of the motor control. In addition to the factors mentioned above, the interindividual scatter may essentially be caused by differences in electrode positioning and by slight deviations of the individual biomechanics of the test persons.

\section{Implications}

Our results show for the first time that the medial pterygoid muscle demonstrates heterogeneous activation for motor tasks simulating forces as they may be generated during bilateral clenching activities of the jaws with various force vectors.

The significant interaction $(p<0.001)$ between the two factors of the two-way ANOVA indicates that the muscle regions react differently to diverse motor tasks, i.e., that they can be activated heterogeneously. Remarkable activity fluctuations with clear reversals of the relative activation could be observed between the anterior and posterior region during anteriorly, medially, and laterally directed forces. This demonstrates that the medial pterygoid has at least two functionally different regions. Due to the multiple compartmentalisation ${ }^{32}$ and the heterogeneous fibre type distribution with distinct 
differences between the anterior and posterior region, ${ }^{14}$ the results do not come unexpected. Nonetheless, the biomechanical relevance of the differential activity distribution during the various motor tasks can only be validly interpreted by understanding the underlying strategies of motor control. Modelling the complex interaction of all masticatory muscles and joint forces on the basis of anatomical geometries, EMG recordings and force measurements represent an instrument to reveal these strategies. ${ }^{33-35}$ To date, it is not known how selective the differential control of the jaw musculature is really in effect. More discrete examination methods may unravel even more than the sub-regions identified in this study. Recordings of individual motor units in the lateral pterygoid muscle point to very selective activation capability within small areas of a muscle, depending on the given motor task. ${ }^{36}$

The general pattern of the EMG amplitudes during the different motor tasks corresponds quantitatively well with the data found in a previous feedback-controlled study with similar clenching directions and forces, but with only one single recording site. ${ }^{30}$ They also match qualitatively with results found in the same investigation for the masseter muscle, demonstrating the close synergism of both muscles during tasks with a relatively pronounced vertical clenching direction.

Furthermore, the results agree with data found for the other masticatory muscles, ${ }^{7,8,21-23}$ and they confirm the heterogeneous activation capability as a general control behaviour for all masticatory muscles. This capacity does not exclude a homogeneous activation, ${ }^{37}$ but it might be an additional control mechanism to meet the requirements for fine motor skills. The possibility to vary the line of action in particular directions might be a favourable strategy particularly during mastication.

Furthermore, the differential motor control makes painful micro-lesions, caused by, for example, repetitive strain, ${ }^{17-20}$ more plausible than for a homogeneously controlled muscle with the same synaptic input. Hence, a differential motor control might favour the development of large regional force vectors (stress concentrations) within the individual muscle. ${ }^{29}$ Indeed, recent studies have confirmed focal lesions in the masseter of rats due to overload. ${ }^{38}$ Finally, it could be shown that positional changes of the mandible, as induced, for instance, by occlusal splints, vary the recruitment patterns in the masticatory muscles. ${ }^{10,39}$ This, in turn, could reduce stress concentrations in particular muscle regions during mastication and clenching and thus unload areas with microlesions. ${ }^{40}$ These implications make the heteroge- neous activation behaviour attractive as an explanatory model for the therapeutic effect of occlusal interventions in patients with temporomandibular disorders. Hence, the model predicts that any positional change (within a physiological range) might be useful for regional pain reduction. This view stands in contrast to the traditional concept of one "physiologically ideal" mandibular repositioning $^{41,42}$ which is still reflected in the various definitions of centric relation. ${ }^{43}$

\section{Acknowledgements}

This investigation was supported by the Deutsche Forschungsgemeinschaft (German Research Society) by grants SCHW 307/15-1 and STRU 675/1-1. The authors wish to express their gratitude to Dr. rer. nat. Maria Pritsch (Institute of Medical Biometry and Informatics, University of Heidelberg) for her statistical consultation.

\section{References}

1. Henneman E, Mendell LM. Functional organisation of motoneuron pool and its inputs. In: Brooks VB., editor. Handbook of physiology. Section I. The nervous system, vol. II, part I. Washington, DC: American Physiological Society; 1981 . p. 423-507.

2. Henneman E, Somjen G, Carpenter DO. Functional significance of cell size in spinal motoneurons. J Neurophysiol 1965;28:560-80.

3. Hoffer JA, Loeb GE, Sugano N, Marks WB, O’Donovan MJ, Pratt CA. Cat hindlimb motoneurons during locomotion. III. Functional segregation in sartorius. J Neurophysiol 1987;57: 554-62.

4. Windhorst U, Hamm TM, Stuart DG. On the function of muscle and reflex partitioning. Behav Brain Sci 1989;12:629-81.

5. Van Zuylen EJ, Gielen CC, Denier van der Gon JJ. Coordination and inhomogeneous activation of human arm muscles during isometric torques. J Neurophysiol 1988;60:152348.

6. Belser UC, Hannam AG. The contribution of the deep fibers of the masseter muscle to selected tooth-clenching and chewing tasks. J Prosthet Dent 1986;56:629-35.

7. Blanksma NG, Van Eijden TM. Electromyographic heterogeneity in the human temporalis muscle. J Dent Res 1990;69:1686-90.

8. Blanksma NG, Van Eijden TM, Weijs WA. Electromyographic heterogeneity in the human masseter muscle. J Dent Res 1992;71:47-52.

9. Schumann NP, Scholle HC, Anders C, Mey E. Mapping spektraler EMG-Parameter des M. masseter bei gesunden Probanden unter definierten Belastungen. EEG EMG Z Elektroenzephalogr Elektromyogr Verwandte Geb 1992;23:121-6.

10. Schindler HJ, Türp JC, Blaser R, Lenz J. Differential activity patterns in the masseter muscle under simulated clenching and grinding forces. J Oral Rehabil 2005;32:552-63.

11. Pratt CA, Chanaud CM, Loeb GE. Functionally complex muscles of the cat hindlimb. IV. Intramuscular distribution of 
movement command signals and cutaneous reflexes in broad, bifunctional thigh muscles. Exp Brain Res 1991;85: 281-99.

12. Stalberg E, Schwartz MS, Thiele B, Schiller HH. The normal motor unit in man. A single fibre EMG multielectrode investigation. J Neurol Sci 1976;27:291-301.

13. McMillan AS, Hannam AG. Motor-unit territory in the human masseter muscle. Arch Oral Biol 1991;36:435-41.

14. Eriksson PO, Thornell LE. Histochemical and morphological muscle-fibre characteristics of the human masseter, the medial pterygoid and the temporal muscles. Arch Oral Biol 1983;28:781-95.

15. Kubota K, Masegi T. Muscle spindle supply to the human jaw muscle. J Dent Res 1977;56:901-9.

16. Rowlerson A, Mascarello F, Barker D, Saed H. Muscle-spindle distribution in relation to the fibre-type composition of masseter in mammals. J Anat 1988;161:37-60.

17. Hägg GM. Static work and myalgia-a new explanation model. In: Andersson PA, Hobart DJ, Danoff JV, editors. Electromyographical kinesiology. Amsterdam: Elsevier Science; 1991. p. 141-4.

18. Armstrong $T J$, Buckle $P$, Fine $L J$, Hagberg $M$, Jonsson $B$, Kilbom $A$, et al. A conceptual model for work-related neck and upper-limb musculoskeletal disorders. Scand J Work Environ Health 1993;19:73-84.

19. Sommerich CM, McGlothlin JD, Marras WS. Occupational risk factors associated with soft tissue disorders of the shoulder: a review of recent investigations in the literature. Ergonomics 1993;36:697-717.

20. Mense S, Simons DG. Muscle pain: understanding its nature, diagnosis, and treatment. Philadelphia: Lippincott Williams \& Wilkins; 2001. p. 253-9.

21. Türp JC, Schindler HJ, Pritsch M, Rong Q. Antero-posterior activity changes in the superficial masseter muscle after exposure to experimental pain. Eur J Oral Sci 2002;110: 83-91.

22. Murray GM, Phanachet I, Klineberg IJ. Electromyographic evidence for functional heterogeneity in the inferior head of the human lateral pterygoid muscle: a preliminary multiunit study. Clin Neurophysiol 1999;110:944-50.

23. Tsuruyama K, Scott G, Widmer CG, Lund JP. Evidence for functional partitioning of the rabbit digastric muscle. Cells Tiss Organs 2002;170:170-82.

24. Scott BJ, Mason AG, Cadden SW. Voluntary and reflex control of the human temporalis muscle. J Oral Rehabil 2002;29: 634-43.

25. Van Eijden TM, Koolstra JH, Brugman P, Weijs WA. A feedback method to determine the three-dimensional bite-force capabilities of the human masticatory system. J Dent Res 1988; $67: 450-4$.

26. Blanksma NG, van Eijden TM. Electromyographic heterogeneity in the human temporalis and masseter muscles during static biting, open/close excursions, and chewing. J Dent Res 1995;74:1318-27.

27. English AW, Weeks OI. Electromyographic cross-talk within a compartmentalized muscle of the cat. J Physiol 1989;416: 327-36.

28. English AW, Carrasco DI, Widmer CG. Torques produced by different compartments of the rabbit masseter muscle. $J$ Appl Biomech 1999;15:348-60.

29. Tonndorf ML, Hannam AG. Motor unit territory in relation to tendons in the human masseter muscle. Muscle Nerve 1994; 17:436-43.

30. Schindler HJ, Rues S, Türp JC, Schweizerhof $\mathrm{K}$, Lenz J. Activity patterns of the masticatory muscles during feedback-controlled simulated clenching activities. Eur J Oral Sci 2005;113:469-78.

31. Trulsson M, Essick GK. Mechanosensation. In: Miles TS, Nauntofte B, Svensson P, editors. Clinical oral physiology. Copenhagen: Quintessence; 2004. p. 165-96.

32. Schumacher GH. Funktionelle Morphologie der Kaumuskulatur. Jena: Fischer; 1961.

33. Koolstra JH, van Eijden TM, Weijs WA, Naeije M. A threedimensional mathematical model of the human masticatory system predicting maximum possible bite forces. $J$ Biomech 1988;21:563-76.

34. Osborn JW. Biomechanical implications of lateral pterygoid contribution to biting and jaw opening in humans. Arch Oral Biol 1995;40:1099-108.

35. Iwasaki LR, Thornton BR, McCall Jr WD, Nickel JC. Individual variations in numerically modeled human muscle and temporomandibular joint forces during static biting. J Orofac Pain 2004; 18:235-45.

36. Murray GM, Phanachet I, Uchida S, Whittle T. The role of the human lateral pterygoid muscle in the control of horizontal jaw movements. J Orofac Pain 2001;15:279-305.

37. Hannam AG, MCMillan AS. Internal organization in the human jaw muscles. Crit Rev Oral Biol Med 1994;5:55-89.

38. Bani D, Bani T, Bergamini M. Morphologic and biochemical changes of the masseter muscles induced by occlusal wear: studies in a rat model. J Dent Res 1999;78:1735-44.

39. Van Eijden TM, Blanksma NG, Brugman P. Amplitude and timing of EMG activity in the human masseter muscle during selected motor tasks. J Dent Res 1993;72:599-606.

40. Türp JC, Schindler HJ. Zum Zusammenhang zwischen Okklusion und Myoarthropathien: Einführung eines integrierenden neurobiologischen Modells. Schweiz Monatsschr Zahnmed 2003;113:964-77.

41. Weinberg LA. Role of condylar position in TMJ dysfunctionpain syndrome. J Prosthet Dent 1979;41:636-43.

42. Dawson PE. Centric relation. Its effect on occluso-muscle harmony. Dent Clin N Am 1979;23:169-80.

43. The Academy of Prosthodontics. The glossary of prosthodontic terms. J Prosthet Dent 2005;94:10-92. 